\title{
A Document / Ein Dokument / \\ Un Document / Un Documento
}

\section{Jung an Freud 1905 : Ein Bericht über Sabina Spielrein}

Bernard Minder

\section{SUMmaRY}

A recently discovered letter, dated September 25, 1905, proves that Jung's first attempt to get in contact with Freud had taken place earlier than it was assumed on the basis of their published correspondence. The letter contains a brief case history of Sabina Spielrein's disease. It was addressed to Freud and handed over to Sabina Spielrein's mother who intended to remove her daughter from Jung with whom the girl had fallen in love. However, the letter was never passed on to Freud.

\section{ZuSAMMENFASSUNG}

Ein neu aufgefundener Brief mit Datum vom 25. September 1905 bezeugt, dass Jung einen ersten Versuch, mit Freud in Verbindung zu treten, früher unternahm, als bisher aus dem publizierten Briefwechsel der beiden bekannt war. Jung verfasste das Schriftstück, das einen kurzen Abriss der Krankheitsentwicklung von Sabina Spielrein enthält, zuhanden der Mutter Spielrein, die ihre 
Tochter aus dessen Behandlung nehmen wollte, weil sie sich in Jung schwärmend verliebt hatte. Frau Spielrein leitete den Brief allerdings nicht an Freud weiter.

\section{Einleitung}

1980 veröffentlichte Carotenuto in Diario di una segreta simmetria - Sabina Spielrein tra Jung e Freud die 1977 in Genf entdeckten Tagebücher und Briefe Sabina Spielreins. 1986 konnte dieses Material in deutscher Erstfassung durch die fehlenden Briefe C. G. Jungs ergänzt werden ${ }^{1}$. Durch diese Publikationen besann man sich auf eine Pionierin der psychoanalytischen Bewegung, die, wie so oft, fast exemplarisch für das Schicksal bedeutender Frauen dieser Zeit, in «Vergessenheit» geraten war.

Sabina Spielrein wurde 1885 in Rostow am Don (Russland) geboren. Sie entwickelte schon in früher Jugend Zeichen einer schweren Hysterie. Als hochintelligentes Mädchen war sie trotz ihres seelischen Zustandes in der Lage, die Schulen erfolgreich zu absolvieren. 1904 wurde sie in desolater Verfassung in die Schweiz gebracht und am 17. August 1904 in die psychiatrische Klinik Burghölzli aufgenommen, wo sie Jungs Patientin bis zu ihrem Austritt am 1. Juni 1905 war. Sie blieb ambulant bis 1909 in Jungs Behandlung. Carotenuto versuchte durch sein veröffentlichtes Material, eine unheilvolle Entwicklung dieser Übertragungs-Gegenübertragungssituation abzuleiten ${ }^{2}$. Der in der vorliegenden Arbeit publizierte Brief vermag zur Klärung der Beziehung Jung-Spielrein beizutragen.

In einer weiteren Beziehung ist dieser Brief bedeutungsvoll. Er dokumentiert Jungs Bestreben, mit Freud in Kontakt zu treten. Nachdem dieser Brief seinen Zweck nicht erfüllte, war es erneut Jung, der durch Zusendung seiner Assoziationsstudien an Freud den Grundstein zu ihrer Zusammenarbeit legte. Der vorliegende Bericht erreichte offensichtlich Freud nie, denn bereits im zweiten Brief Jungs an Freud vom 23. Oktober 1906 bringt dieser erneut einen kurzen Abriss der Krankheitsentwicklung Sabina Spielreins vor $^{3}$.

Dieser von C.G. Jung signierte Brief fand sich als Durchschlag in den «Kopierbüchern» der Psychiatrischen Universitätsklinik Burghölzli. Seit 1878 (Klinikeröffnung 1870) wurden sämtliche Briefe der ärztlichen Direktion, die das Haus verliessen, als Doppel archiviert; sie sind als solche 
gebunden bis 1936 vollständig erhalten. Sie enthalten die Korrespondenz bezüglich der hospitalisierten Patientinnen und Patienten. Selten taucht ein Brief privaten Charakters auf. Von den Briefzugängen zur Klinik sind leider nur sehr vereinzelte erhalten geblieben. Obiger Brief ist uns aus der Arbeit Carotenutos zum Teil bekannt, das Datum wird jedoch als «Irrtum» bezeichnet mit dem Hinweis, dass der Briefwechsel Jung-Freud erst am 11.4.1906 seinen dokumentierten Anfang nehme.

Für die Publikationserlaubnis danke ich den Nachfahren Sabina Spielreins und der ärztlichen Direktion der Klinik Burghölzli (Prof. Dr. D. Hell).

\section{Der Brief («Bericht»)}

Quelle: Kopierbücher Burghölzli, Band 63; Burghölzli-Archiv.

Buchstaben-, jedoch nicht zeilengetreue Transkription.

$\ll 25.9 .1905$

Bericht über Fräulein Spielrein an Herrn Professor Freud in Wien, an Frau Spielrein übergeben zu eventueller Verwendung.

Sehr geehrter Herr Professor,

Die Tochter von Frau Spielrein, Fräulein Sabina Spielrein stud.med. leidet an Hysterie.

Pat. ist erblich erheblich belastet, Vater \& Mutter sind hysterisch, besonders die Mutter. Ein Bruder der Pat. ist seit frühester Jugend schwer hysterisch.

Pat. wird jetzt 20 Jahre, vor ca 3 Jahren ist die Krankheit deutlich zum Vorschein gekommen. Die pathogenen Erlebnisse reichen aber natürlich weit in die Jugend zurück. Ich habe nach Ihrer Methode das Krankheitsbild ziemlich vollständig analysiert, auch mit Anfangs sehr günstigem Erfolg.

Die Analyse hat im Wesentlichen ergeben:

Die körperlichen Züchtigungen auf den Hintern, die der Vater der Pat. zwischen dem $4 \&$ \& ten [Lebensjahr] appliciert hat, haben sich unglücklicherweise mit dem praematuren \& jetzt sehr starken Sexualgefühl der Pat. associiert. Die Sexualität aeusserte sich schon sehr frühe darinn, dass Pat. durch Aneinanderpressen der Oberschenkel zu masturbieren anfieng. Die Masturbation trat immer auf nach Züchtigungen die sie durch den Vater erlitt. Allmählig waren zur Hervorrufung der sexuellen Erregung nicht mehr 
Schläge nöthig, sondern sie trat auch auf blosse Drohungen ein, auch auf bloss sonst etwas gewaltsame Situationen, z. B. Schimpfworte, drohende Handbewegungen etc. Sie konnte schliesslich die Hände des Vaters nicht mehr ansehen, ohne sexuell erregt zu werden, sie konnte ihn nicht mehr essen sehen, weil sie dabei denken musste, wie das Essen wieder hinausgeht, dann Züchtigung der Nates etc. Diese Associationen dehnten sich auch auf den jüngeren Bruder aus, der ebenfalls seit langem stark onaniert. Drohungen oder kleine Misshandlungen des Knaben erregten sie, ebenso musste sie masturbieren, wenn sie sah, wie er gezüchtigt wurde. Allmählig erregten sie alle, nur irgendwie an etwas Gewaltsames errinnernde Situationen, z.B. wenn ihr jemand sagte, sie müsse gehorchen. Sobald sie alleine war, wurde sie von Zwangsvorstellungen geplagt, z. B. musste sie sich alle möglichen Quälereien vorstellen, das gleiche $\mathrm{g}[\mathrm{e}] \mathrm{sch}$ ah auch in ihren Träumen, so träumte sie z. B. häufig, sie esse zu Mittag \& sitze dazu auf dem Locus \& alles gehe gleich wieder hinten hinaus, dazu befand sich um sie eine grosse Menschenmenge, welche ihr zusah; ein andermal wurde sie vor einer grossen Volksmenge ausgepeitscht. etc.

Dadurch wurde natürlich ihr Zustand zu Hause unhaltbar. Nach vielen grossen Aufregungen wurde sie vor ca 1 Jahre in die Schweiz gebracht, zuerst in ein Sanatorium, wo aber der Arzt ihren geradezu dämonischen Launen \& Chicanen durchaus nicht gewachsen war. Sie brachte dort alle Leute zur Verzweiflung. Schliesslich gieng es in der Privatanstalt nicht mehr \& wurde zu uns in die Irrenanstalt gebracht. Hier war sie am Anfang furchtbar chicanös, quälte die Pflegerinnen bis aufs Blut. Mit fortschreitender Analyse besserte sich ihr Zustand zusehends \& schliesslich entpuppte sie sich als eine höchst intelligente \& begabte Person von grösster Sensibilität. Ihr Charakter hat entschieden etwas rücksichtsloses \& unbilliges, auch fehlt jegliches Gefühl für Opportunität \& äusseren Anstand, wovon viel natürlich auf russische Eigenthümlichkeit muss geschoben werden.

Ihr Zustand besserte sich so beträchtlich, dass sie letztes Sommersemester studieren konnte. Sie leidet natürlich sehr darunter, wenn sie immer wieder mit ihren Angehörigen zusammenkommt, was besonders die Mutter nicht recht begreifen will, aber aus allem obgesagtem sehr wohl verständlich ist. (Frau Spielrein kennt übrigens den wichtigsten Theil des Complexes ihrer Tochter.)

Während der Behandlung hatte die Pat. die Malchance, sich in mich zu verlieben. Sie schwärmt nun der Mutter immer in ostentativer Weise von ihrer Liebe vor, wobei eine geheime chicanöse Freude am Schrecken der 
Mutter eine nicht unbeträchtliche Rolle spielt. Die Mutter will sie desshalb nun, im Falle der Noth, in eine andere Behandlung geben, womit ich natürlich einverstanden bin.

Mit vorzüglicher Hochachtung

Ihr ergebenster

Dr. Jung»

\section{Diskussion}

Das Datum des Schriftstückes ist in mehrerer Hinsicht höchst bemerkenswert. Zum einen handelt es sich hier um den allerersten Kontaktversuch Jungs zu Freud. Zum andern wurde der Brief zu einer Zeit auf Papier der ärztlichen Direktion verfasst, als Sabina Spielrein schon drei Monate nicht mehr Patientin am Burghölzli war. Was veranlasste wohl Jung, für seine ambulante Patientin das Papier der ärztlichen Direktion zu verwenden? Die einfachste Erklärung liegt wohl in folgenden Umständen. Jung bekleidete seit April 1905 die Stellung eines Sekundararztes; damit hatte er bei Abwesenheit von Prof. Eugen Bleuler dessen Vertretung zu übernehmen. Dies war Ende September 1905 der Fall ${ }^{4}$. Wie wir dem Brief entnehmen, suchte die Mutter Spielrein Jung auf, um ihre Tochter in eine «andere Behandlung» zu geben. Sie fand Jung also in der Klinik, und dieser benutzte naheliegenderweise das vorhandene Schreibpapier. Dieser Punkt ist deshalb wichtig, weil die Verwendung von Klinik-Briefpapier für ambulante Patienten einmalig zu sein scheint, wie die Durchsicht sämtlicher von Jung signierter Briefe im Klinikarchiv ergibt.

Tituliert ist das Schreiben als «Bericht». Es handelt sich also nicht um ein ärztliches Überweisungsschreiben im engeren Sinne, was durch den Passus «zu eventueller Verwendung» verdeutlicht wird. Letztere Redewendung gibt zur Vermutung Anlass, dass sich die Mutter Spielrein Jung gegenüber in einer unbequemen Situation befand. Immerhin hatte die Betreuung der Tochter durch Jung während der gut neunmonatigen Hospitalisation am Burghölzli zu einer dramatischen Besserung geführt. Sie konnte nach sechs Monaten an der Klinik ihr Studium in Medizin aufnehmen. Nun musste sie im «Falle der Noth» die Tochter vor einer angeblichen Liebesbeziehung bewahren. Sicher war sie wohl nicht, denn sie wollte dieses Schreiben nur «eventuell» verwenden. Warum Sabina Spielrein letztendlich in Jungs Be- 
handlung blieb, ist nicht zu beantworten. Jedenfalls wurde sie nie Freuds Patientin.

Als nächstes stellt sich die Frage, warum sie ausgerechnet an Freud nach Wien hätte überwiesen werden sollen. Immerhin wohnte Sabina Spielrein in Zürich und studierte da. Möglich ist, dass dies einem Wunsch der Mutter entsprach. Eine andere, viel plausiblere Erklärung bietet folgender Zusammenhang: Jung schreibt:

«Ich habe nach Ihrer Methode das Krankheitsbild ziemlich vollständig analysiert, auch mit Anfangs sehr günstigem Erfolg.»

Sabina Spielrein war die erste Hysterie-Patientin Jungs, an der er die Freudsche «Methode» angewandt hatte (vgl. Anmerkung 2). Es lag also einerseits im Sinne der Patientin (in Bezug auf die Behandlungskontinuität) nahe, sich an Freud zu wenden, andererseits konnte Jung mit Sabina Spielrein gleich zu Beginn eines von ihm erhofften Kontakts zu Freud mit einem interessanten Fall aufwarten. Später war es erneut Jung, der durch Zusendung seiner Assoziationsstudien den Grundstein einer gegenseitig nützlichen und fruchtbaren Beziehung legte (vgl. den ersten Brief Freuds an Jung; McGuire 1974).

Jungs «Bericht» beginnt mit dem sehr ungewöhnlichen Vermerk, dass dieser der Mutter Spielrein übergeben wurde. Jung ordnete offenbar die ärztliche Schweigepflicht der elterlichen Gewalt unter. (Die Patientin war zu diesem Zeitpunkt noch nicht ganz 20jährig.) Es folgen einige Hinweise auf die familiäre Belastung, ein kurzer Abriss der Krankheitsentwicklung und des therapeutischen Ansatzes (Hinweise auf Arbeit mit Traummaterial) sowie über den Verlauf. Damit bestätigt sich weitgehend die uns durch Carotenutos Publikation bekannte Psychopathologie. Neu dabei ist, dass Jung sich an den Ansätzen Freuds zur Dynamik der Hysterie-Genese orientierte. Erstaunlich ist hier - wie auch in der späteren Korrespondenz zwischen Jung und Freud - die vermeintliche Erkenntnis, dass sich die «körperlichen Züchtigungen auf den Hintern [...] unglücklicherweise mit dem prämaturen \& jetzt sehr starken Sexualgefühl der Pat. associiert» hätten. Ein «Trauma» wird also mit einem «Sexualgefühl» assoziiert, es ist keine Rede von «sexuellem Trauma». Die Inzestproblematik wird auch später nie diskutiert. Mit welcher Unsicherheit sich Jung verständlicherweise auf diesem Gebiet bewegte, wird einerseits dadurch verdeutlicht, dass er die Patientin lediglich «ziemlich vollständig» analysiert hatte, andererseits durch die etwas unbeholfene Formulierung über ihren «Charakter» : «[...] wovon viel 
natürlich auf russische Eigenthümlichkeit muss geschoben werden.» Man beachte auch die Wortwahl «geschoben», die hier stellvertretend für «noch ungelöste Probleme vor sich herschieben» zu sehen ist. Befremdend wirkt dagegen die Behauptung, dass Rücksichtslosigkeit, Unbilligkeit und fehlendes Gefühl für Opportunität und äusseren Anstand russische Eigentümlichkeiten sein sollen.

Am Ende des «Berichtes» wird schliesslich die Frage beantwortet, wieso dieser überhaupt abgefasst wurde. Die Patientin hatte die «Malchance», sich in Jung zu verlieben und agierte offensichtlich damit unter «geheime[r] chicanöse[r] Freude am Schrecken der Mutter». Wie wir aus Jungs Publikation Assoziation, Traum und hysterisches Symptom (1906) wissen ${ }^{5}$, war für ihn diese Situation nicht neu. In der erwähnten Arbeit berichtet er über ähnliche Gefühlsregungen zweier Patientinnen, die im Frühjahr/Sommer 1905 im Burghölzli hospitalisiert waren (wie die Durchsicht der Krankenakten ergibt), also noch vor dem Vorsprechen der Mutter Spielrein. Die in dieser Publikation hauptsächlich besprochene Patientin wurde dem Assoziationsexperiment unterzogen und die dabei gewonnenen Erkenntnisse den Analysen ihrer Träume gegenübergestellt. Aus dieser Arbeit möchte ich aus zwei Traumanalysen zitieren, Traum Nr. 5 bzw. 7 (S.414 bzw. 417):

«Der schwarze Mann verwandelt sich hier in die weisse Gestalt, das brennende Haus ist der Sexualkomplex. Fräulein L. [die andere Kranke; Verfasser] ist eine Patientin, welche für den Referenten schwärmt. Sie ist infolge eines erotischen Komplexes krank geworden, wie die Patientin. Die Patientin drückt daher durch diese Person aus, dass sie sich in den Referenten verliebt hat. Damit ersetzt die Patientin das ihrer Energie schädliche Zärtlichkeitsverhältnis zur Mutter durch die erotische Beziehung zum Arzt».

«Sie möchte also mit dem Arzte in sexuelle Verbindung treten. [...] dazu kommt aber der offenbar tiefe Eindruck, den ihr meine frühere Analyse machte, in der ich ihre Illusionen schonungslos zerstörte.»

Jung wusste also um das Phänomen der Übertragungsliebe und vermochte dieses analytisch(-therapeutisch) umzusetzen. Eine klare Abgrenzung diesen Patientinnen gegenüber war ihm offenbar möglich. Dass er aber dann im Zusammenhang mit Sabina Spielrein von «Malchance» spricht, ist bemerkenswert. An dieser Stelle ist auch der Umstand zu erwähnen, dass Sabina Spielrein in ihrer Krankheitsentwicklung mehrfach «Opfer» solcher Gefühlsregungen war. Ob Jung letztlich unter dem Druck der Mutter oder aufgrund einer Ahnung über die kommende Entwicklung seiner Beziehung zur Patientin sich «natürlich einverstanden» erklärte, sie in eine «andere Behandlung» zu geben, muss hier offen bleiben. 


\section{Anmerkungen}

1 Carotenuto, A., Tagebuch einer heimlichen Symmetrie. Kore Verlag, Freiburg i. Br. 1986.

2 Bis heute war die Zeit ihrer Hospitalisation im dunkeln geblieben. Im Rahmen meiner Dissertation (1992) ist die vollständige Krankenakte veröffentlicht. Dabei wird die Bedeutung des «Falles Spielrein» für Jung und die Klinik erläutert.

3 McGuire, W., Sigmund Freud-C. G. Jung, Briefwechsel. Fischer, Frankfurt am Main 1974.

4 Antrag Bleulers an den Regierungsrat vom 28.8. 1905 für Urlaub vom 7.9. bis 28. 9. 1905, Rückmeldung Bleulers am 28.9.1905; Burghölzli-Archive.

5 Jung, C.G., Gesammelte Werke, Band 2 (2. Auflage). Walter, Olten 1979.

Bernard Minder, Dr. med.

Psychiatrische Universitätsklinik Burghölzli

Postfach

8029 Zürich 